\section{Abordaje citogenético y citogenómico de pacientes con discapacidad intelectual y malformaciones congénitas}

\section{Cytogenetic and Cytogenomic Approach of patients with Intellectual Disability and Congenital Malformations.}

Yokoyama-Rebollar $\mathrm{E}^{1}$, Frías $\mathrm{S}^{2,3}$, Del Castillo-Ruiz $\mathrm{V}^{1}$

\section{DEFINICIONES}

La discapacidad intelectual (DI) o retraso mental tiene una prevalencia del $2-3 \%$ en la población general y se define como una alteración del neurodesarrollo que inicia antes de los 18 años. Se caracteriza por limitación importante en el funcionamiento intelectual y en el comportamiento adaptativo en áreas como comunicación y uso de fuentes para la misma, autocuidado, relaciones sociales o interpersonales, autodirección, funciones académicas, salud y seguridad. ${ }^{1,2}$ La DI se determina por un coeficiente intelectual $(\mathrm{Cl})$ menor de 70 puntos mediante escalas como la International Classification of Diseases (ICD-10), Diagnostic and Statistical Manual of Mental Disorders (DSM-V) y la clasificación World Health Organization (WHO). ${ }^{3,4}$

En los pacientes menores de 5 años se considera como retraso global del desarrollo o retraso psicomotor (RPM) y para su definición se utilizan diferentes escalas como la de Gesell, la cual

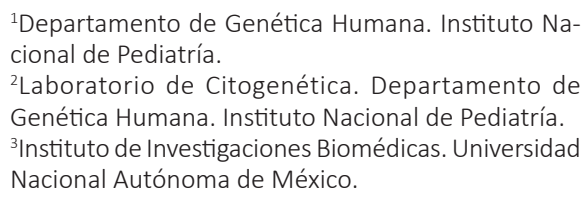

${ }^{1}$ Departamento de Genética Humana. Instituto Nacional de Pediatría.

${ }^{2}$ Laboratorio de Citogenética. Departamento de Genética Humana. Instituto Nacional de Pediatría. ${ }^{3}$ Instituto de Investigaciones Biomédicas. Universidad Nacional Autónoma de México.

Recibido: 18 de septiembre del 2017

Aceptado: 19 de septiembre del 2017

Correspondencia

Dra. Emiy Yokoyama Rebollar eyr75@hotmail.com

Este artículo debe citarse como Yokoyama-Rebollar E, Frías S, Del Castillo-Ruiz V. Abordaje citogenético y citogenómico de pacientes con discapacidad intelectual y malformaciones congénitas. Acta Pediatr Mex. 2017;38(6):433-441. 
otorga un porcentaje del desarrollo mediante la evaluación de las áreas de lenguaje, motricidad, psicosocial y adaptativo. ${ }^{5}$ El promedio del $\mathrm{Cl}$ en la población general es de 100, limítrofe entre 70 y 85 y por debajo de 70 se considera DI. Un CI de 50-70 es DI leve, moderado de 35-49, grave de 20-34 y profundo cuando es menor de 20. El 2 al $2.5 \%$ de la población tiene DI leve y sólo 0.3 a $0.5 \%$ lo presenta grave y de estos últimos del 25 al 50\% son de etiología genética y suelen acompañarse de otras manifestaciones clínicas. ${ }^{6}$

Por lo general, los pacientes con RPM o DI presentan múltiples malformaciones congénitas asociadas, las cuales se definen como anomalías morfológicas que se originan antes de nacer, se presentan en un 3\% de los recién nacidos, aunque esta cifra se duplica en el transcurso de la vida debido a la presencia de defectos no detectables al nacimiento y contribuyen de manera importante a pérdidas gestacionales y a la morbimortalidad pediátrica. ${ }^{7,8}$

\section{ETIOLOGÍA}

Es bien conocido que la DI es de origen multifactorial, aunque también puede haber causas solo ambientales o genéticas que lo condicionen. A nivel ambiental destacan la desnutrición durante el embarazo, exposición a agentes físicos (radiaciones), químicos (alcoholismo materno) o biológicos (TORCH). ${ }^{5}$ En la etiología genética se encuentran: a) Las alteraciones cromosómicas tanto numéricas como estructurales, en donde encontramos aneuploidías como trisomías 13, 18 y 21, síndromes de Klinefelter y Turner con sus variantes, así como translocaciones no balanceadas, deleciones, duplicaciones y rearreglos complejos; todos éstos implican un desbalance en el número de copias de regiones génicas que pueden ser identificadas por medio de Citogenética o Citogenómica; b) Las alteraciones monogénicas, de las cuales existen más de 1200 entidades con DI que están referidas en el sistema de Online Mendelian Inheritance in Man (OMIM - www.ncbi.nlm.nih.gov/sites/ entrez?db=omim) y aunque se sabe que los genes relacionados con la función cerebral se encuentran distribuidos a lo largo de todo el genoma, actualmente se ha descrito que el número de genes relacionados con la discapacidad intelectual es mucho mayor en el cromosoma $\mathrm{X}$ que en cualquier otro cromosoma. ${ }^{9}$

En los últimos años, se han identificado varios genes como el FMR2, OPHN1, GDI1, PAK3, IL1RAPL, TM4SF2, VCX-A, y ARHGEF6, los cuales también pueden ser parte de la etiología del RPM o DI sindrómico, autismo u otros defectos del neurodesarrollo. ${ }^{10}$

El presente trabajo se enfoca en la primera parte de la etiología genética, relativo al desbalance en el genoma, ya sea por cambio en el número de cromosomas o de número de copias de regiones subcromosómicas.

\section{ABORDAJE}

Clínico

Actualmente, las causas de la DI son mejor conocidas; hasta la fecha se sabe que sólo en el 40 a $60 \%$ de los pacientes con DI se conoce la causa genética, de los cuales este porcentaje es menor cuando los pacientes presentan DI leve o moderado. ${ }^{11}$ Por esto, para conocer la causa de un cuadro clínico con DI y dismorfias se requiere realizar un abordaje integral del paciente que comprende una historia clínica completa y detallada con árbol genealógico, antecedentes familiares, antecedentes prenatales, perinatales y evolución postnatal, con una exploración física minuciosa de dismorfias mayores y menores, exploración neurológica y evaluación mental. ${ }^{12}$

Dentro de los estudios de laboratorio y gabinete que se solicitan están las pruebas de función 
Yokoyama-Rebollar E et al. Abordaje de pacientes con DI y malformaciones

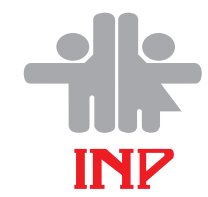

tiroidea, TORCH (por sus iniciales en inglés de toxoplasmosis, rubeola, citomegalovirus, herpes simple y VIH), tamiz metabólico y cariotipo, así como ultrasonido (USG) transfontanelar, tomografía axial computada (TAC), o en su caso resonancia magnética (IRM) cerebrales. También es importante tomar en cuenta la utilidad de las bases de datos para diagnóstico como el Pictures Of Standard Syndromes and Undiagnosed Malformations (POSSUM) y el London Dysmorphology Database.

El abordaje idóneo para el estudio de los pacientes con DI se ilustra en la Figura 1, la cual nos muestra las rutas adecuadas para tratar de llegar a un diagnóstico etiológico; sin embargo, es importante recalcar que esto se logra solo en el $50 \%$ de los casos con DI. ${ }^{9}$

\section{Citogenético}

El número de 46 cromosomas en la especie humana fue descubierto en $1956,{ }^{13}$ y las aneuploidías en 1959. Cuando Lejeune y cols. ${ }^{14}$ reportaron que el síndrome de Down se debía a la trisomía de un cromosoma 21 (OMIM 190685) y que era la causa más común del retraso mental. La introducción de técnicas como la de bandas G en los 70s, facilitó la identificación de cada uno de los cromosomas, así como la detección de alteraciones cromosómicas relacionadas con algunos fenotipos, ${ }^{15}$ de manera que se integraron síndromes que cursan con DI y malformaciones y que pueden ser distinguibles clínicamente y comprobables con un cariotipo convencional, como las aneuploidías más frecuentes, las trisomías 13, 18, 21, y de cromosomas sexuales.

Otras alteraciones cromosómicas estructurales, como algunas deleciones o duplicaciones detectables al microscopio, se han podido integrar como una entidad reconocible por sus características fenotípicas. Las deleciones de los cromosomas $5 p$ (síndrome de Cri-du-chat) o 4p (síndrome de Wolf) son detectables mediante el cariotipo con bandeo G y generalmente resuelve el diagnóstico. En pacientes sin un síndrome identificable, pero con DI y dismorfias, se han detectado alteraciones en prácticamente todos los cromosomas y generalmente desbalanceadas; las más frecuentes son las translocaciones, deleciones, duplicaciones e inversiones.

Las frecuencias reportadas de alteraciones cromosómicas detectadas al microscopio son muy variables. Xu y cols. ${ }^{16}$ reportaron una media de 16.2\% (rango: $4-34.1 \%$ ), Shevell y cols. ${ }^{17}$ un 3.7\% (rango: $2.9-11.6 \%$ ) y en la revisión más reciente de van Karnebeek y cols. ${ }^{18}$ la media fue de 9.5\% (rango: 0-48.5\%). De esta manera, el cariotipo convencional con bandeo G es una metodología relativamente barata y accesible, su resolución a 400-500 bandas (lo más utilizado), permite detectar alteraciones cromosómicas mayores a 5 o 10 megabases (Mb) y esto nos permite proporcionar un diagnóstico de certeza en el $10 \%$ de los pacientes con sospecha de cromosomopatía (Figura 2). ${ }^{19}$

Entre 1980 y 1990 se incrementó la resolución del estudio citogenético al utilizar la metodología de hibridación in situ con fluorescencia (FISH), la cual usa sondas de DNA marcadas con fluorocromos, para identificar la presencia, el número y localización de regiones cromosómicas pequeñas (sub-microscópicas) y así detectar microdeleciones, microduplicaciones y translocaciones crípticas (Figura 3). Para realizar el FISH se requiere tener la información o la sospecha clínica del segmento cromosómico involucrado, debido a que el estudio no es a nivel genómico, sino totalmente dirigido a una región específica del genoma. Esta metodología es muy útil para el diagnóstico de microdeleciones como la 22q11 o 1p36, ya que en estos pacientes el fenotipo puede hacer sospechar la alteración cromosómica y la resolución del FISH al ser alta puede detectar alteraciones tan pequeñas como de kilobases utilizando las sondas apropiadas. Actualmente existe una larga 
Paciente con retraso mental y malformaciones congénitas

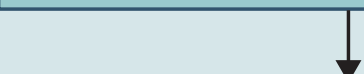

1. Historia clínica detallada con árbol genealógico.

- Antecedentes familiares.

- Antecedentes prenatales y perinatales.

- Evolución posnatal.

2. Exploración física minuciosa.

- Determinación de dismorfias mayores y menores.

3. Evaluación psicológica.

- Determinación de coeficiente global del desarrollo.

- Determinación de coeficiente intelectual.

4. Evaluación por otros servicios dependiendo de los hallazgos clínicos encontrados.

encontrados.

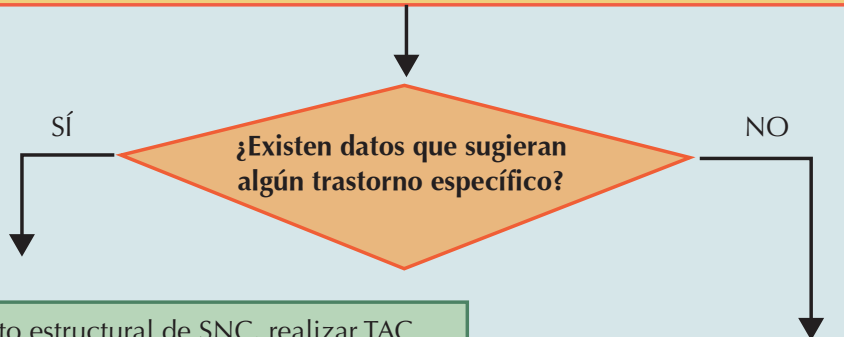

1. Sospecha de defecto estructural de SNC, realizar TAC o USGT, o de disgenesia cerebral, realizar RMN.

2.Sospecha de error innato del metabolismo, tamiz metabólico.

3. Sospecha hipotiroidismo, pruebas de función tiroidea. 4. Sospecha de defecto cromosómico, realizar estudio de citogenética.

5. Sospecha síndrome de X-frágil o síndrome de Rett se realiza estudio molecular específico.

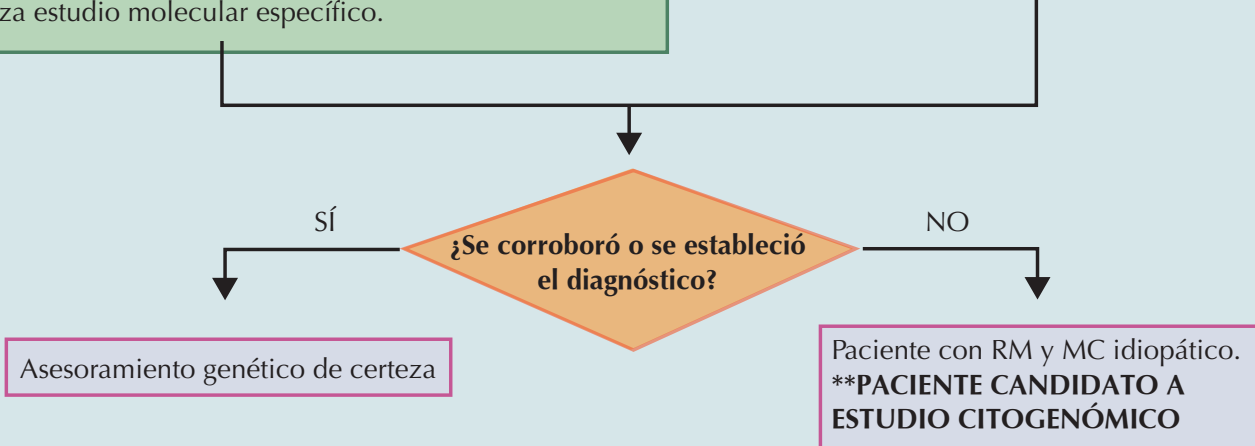

SNC: sistema nervioso central; TAC: tomografía axial computada; USGT: ultrasonido transfontanelar; RMN: resonancia magnética nuclear; DI: discapacidad intelectual; MC: malformaciones congénitas.

Figura 1. Algoritmo del abordaje de pacientes con DI y MC. 


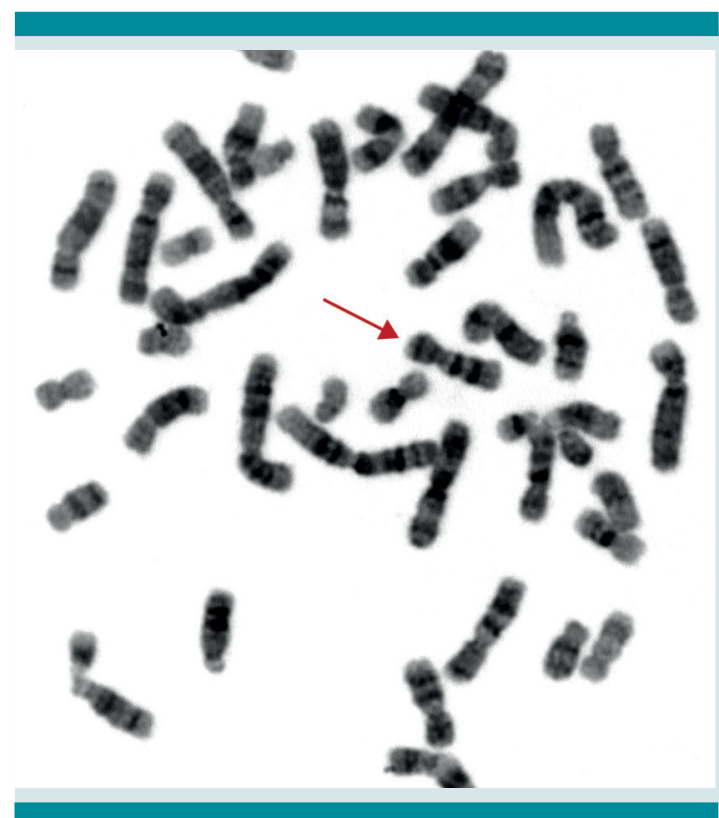

Figura 2. Cariotipo con deleción 7p15: 46,XY, del(7) (p15.2).

lista de microdeleciones o microduplicaciones que se han integrado como síndromes y son reconocibles por la experiencia clínica de los médicos tratantes (Cuadro 1).

\section{Citogenómico}

Cuando los pacientes con DI y dismorfias no tienen un fenotipo que haga sospechar de alteraciones en regiones cromosómicas específicas, se debe recurrir a metodologías que exploren el genoma completo. Por esto, se desarrolló otra metodología de mayor resolución (3-5Mb) conocida como hibridación genómica comparativa $(\mathrm{CGH})$. Ésta originalmente utilizaba como sonda el genoma completo y como blanco metafases normales completas, sin embargo, la capacidad de detección de alteraciones era todavía a nivel de cromosomas, $^{8}$ por lo que rápidamente se migró al desarrollo de metodologías de microarreglos de $\mathrm{CGH}(\mathrm{aCGH}),{ }^{8}$ los cuales tuvieron sus inicios en 1997 con Salinas-Toldo y cols., ${ }^{20}$ así como en 1998 con Pinkel y cols. ${ }^{21}$ Los aCGH cuentan con una resolución $\leq 1-\mathrm{Mb}$, ya que en lugar de cromosomas metafásicos utiliza segmentos de DNA obtenidos de clonas de DNA de regiones conocidas del genoma humano u oligonucleótidos, que están fijos sobre una laminilla y cuyo sitio en el genoma humano se

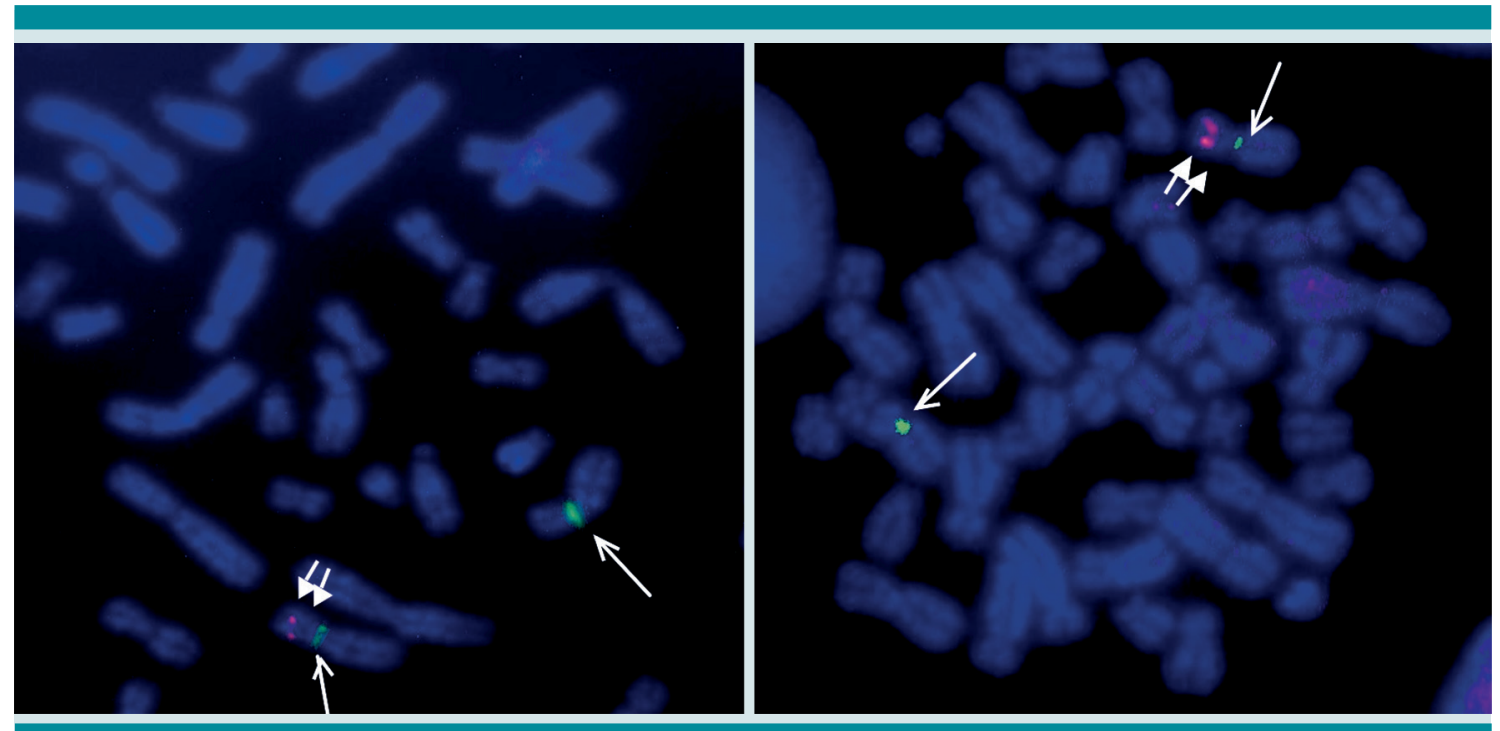

Figura 3. Análisis de FISH para la detección de una microdeleción 7p15. Análisis de una metafase con las sondas alfa satélite (una flecha delgada), para la localización del centrómero del cromosoma 7 (D7Z1) marcada con fluorocromo verde (Q-BIOgene) y sonda locus específica (dos flechas gruesas) para la detección de la región 7p15.2 en donde se localizan los genes HOXA6-HOXA13 marcada con fluorocromo rojo (SureFiSH, Agilent). 
Cuadro 1. Síndromes por microdeleción y por microduplicación

\begin{tabular}{l|c|c|c|}
\hline Síndromes por microdeleción & $\begin{array}{c}\text { Región } \\
\text { involucrada }\end{array}$ & Síndromes por microduplicación & $\begin{array}{c}\text { Región } \\
\text { involucrada }\end{array}$ \\
\hline Deleción 1p36 & $1 \mathrm{p} 36.33$ & Síndrome de duplicación $2 q 31.1$ & $2 q 31.1$ \\
\hline Síndrome de Williams & $7 q 11.23$ & Síndrome de duplicación $2 q 35$ & $2 q 35$ \\
\hline Síndrome de Langer-Giedion & $8 q 24.1$ & Síndrome de duplicación 3q29 & $3 q 29$ \\
\hline $\begin{array}{l}\text { Síndrome de WAGR (Tumor de Wilms, } \\
\text { aniridia, alteraciones de genitales, retraso } \\
\text { mental) }\end{array}$ & $11 p 13$ & Síndrome de triplicación WBS (Williams- & $7 q 11.23$ \\
\hline Síndrome de Angelman & $15 q 11.2$ & Seuren Syndrome) 7q11.23 & $8 q 22.1$ \\
\hline Síndrome de Prader Willi & $15 q 11.2$ & Síndrome de duplicación 15q11q13 & $15 q 11 q 13$ \\
\hline Síndrome de Rubinstein-Taybi & $16 p 13.3$ & Síndrome de Potocki-Lupski & $17 p 11.2$ \\
\hline Síndrome de Smith Magenis & $17 p 11.2$ & Charcot-Marie-Tooth1A & $17 p 11.2 p 12$ \\
\hline Síndrome Miller-Dieker & $17 p 13.3$ & Síndrome de duplicación 17q12 & $17 q 12$ \\
\hline Síndrome Velocardiofacial & $22 q 11.2$ & Síndrome de duplicación 17q21.31 & $17 q 21.31$ \\
\hline Síndrome Phelan-Mcdermid & $22 q 13.33$ & Síndrome de duplicación 22q11.2 & $22 q 11.2$ \\
\hline Síndrome de Kallmann & Xp22.31 & Síndrome de duplicación Xq25 & Xq25
\end{tabular}

conoce muy bien. Por lo general, estos oligonucleótidos del genoma completo se tienen por triplicado en el microarreglo y se procesan en plataformas comerciales. Esta metodología se fundamenta también en la hibridación genómica comparativa, en la cual se utiliza como sonda el genoma completo del paciente, el DNA se fragmenta y se marca con un fluoróforo verde (Cianina 3 o Cy3) y de manera paralela se utiliza un DNA de referencia para la comparación; el genoma de referencia puede provenir de un pool de DNAs de personas normales que se fragmenta y se marca con un fluoróforo rojo (Cianina 5 o Cy5); una vez marcados, los dos DNAs en relación 1:1 se hibridan competitivamente sobre el microarreglo, de manera que si la relación Cy3:Cy5 está alterada, indica una pérdida o una ganancia del DNA del paciente. ${ }^{22}$

Los aCGH permiten el escaneo completo del genoma, lo que da lugar a la detección de variaciones en el número de copias (CNVs), las cuales forman parte de la evolución del ser humano y de la diversidad genética entre individuos, pero también se han encontrado CNVs que son causales de diversas enfermedades que incluyen DI. ${ }^{23}$ Dentro de las limitantes de esta tecnología, se encuentra la incapacidad de detectar alteraciones cromosómicas balanceadas como translocaciones o inversiones y tampoco puede detectar mosaicos bajos (<20\%). La interpretación de los resultados requiere de un análisis muy preciso utilizando diversas bases de datos internacionales como Database of Genomic Variants (DGV), International Standard Cytogenomic Array consortium Databases (ISCA), Database of Chromosomal Imbalance and Phenotype in Humans using Ensemble Resources (DECIPHER) y Online Mendelian Inheritance in Man (OMIM). Este análisis requiere de la experiencia del Médico Genetista para poder asociar las características fenotípicas con las funciones de los genes que se encuentran en dosis anormales, así como para la interpretación de CNVs que involucran genes con función aún desconocida, y de manera muy importante, para identificar variantes normales (polimorfismos), no relacionados con el cuadro clínico del paciente. Este análisis constituye el 
Yokoyama-Rebollar E et al. Abordaje de pacientes con DI y malformaciones

paso definitivo para detectar las alteraciones genómicas causales de la enfermedad, como desbalances por microdeleciones o por microduplicaciones, posibles cromosomas derivativos, aneuploidías y rearreglos subteloméricos. ${ }^{24-27}$

En las alteraciones por microdeleción y/o microduplicación el espectro del fenotipo, definido como las características bioquímicas, fisiológicas y morfológicas observadas en un individuo, determinadas por el genotipo y el ambiente en el que se expresa, se puede atribuir al mal funcionamiento de un gen sensible a dosis, ya sea por reducción de la cantidad de proteína secundaria a la pérdida del gen o por ganancia de función como consecuencia de una duplicación de un gen o genes completos.

En los síndromes de genes contiguos, generalmente el fenotipo resulta de la inapropiada dosis génica de una región determinada; el fenotipo en ciertas ocasiones es fácilmente reconocible por las características clínicas, lo que permite tener una sospecha diagnóstica (ej. síndrome de Prader-Willi, síndrome de Williams); sin embargo, en muchas otras ocasiones esto resulta difícil, a pesar de la fuerte asociación bien conocida entre DI, malformaciones congénitas y dismorfias faciales con desbalances genómicos.

Actualmente, además de estudios en CNVs, existe el análisis de microRNAs (miRNAs) en pacientes con discapacidad intelectual o retraso mental. Los miRNAs son RNAs cortos (de 20 a 23 nucléotidos) no codificantes que regulan la expresión de ciertos genes a nivel postranscripcional. Se sabe que más del $90 \%$ de los genes humanos se regulan por miRNAs e incluso, alrededor del $70 \%$ de estos pequeños RNAs se expresan en cerebro y tienen función en el neurodesarrollo, neurotransmisión, plasticidad sináptica y crecimiento neuronal. En un estudio reciente sobre miRNAs en población con DI, se demostró un incremento significativo del involucro de éstos en los CNVs patogénicos reportados en el DECIPHER. Así mismo, los miRNAs encontrados en los CNVs de novo, tienen mayor expresión en tejidos cerebrales en comparación con los de los CNVs comunes, por lo que los autores sugieren que los miRNAs de los CNVs de novo y de los patogénicos pudieran contribuir a la etiopatogenia de la DI. ${ }^{28}$

Diversos estudios con aCGH demuestran una variabilidad en la frecuencia de detección de alteraciones cromosómicas (Cuadro 2); ;9,10,30${ }^{34}$ sin embargo, el porcentaje en el grupo de pacientes con RPM o DI asociado o no con malformaciones congénitas y con cariotipo normal continúa siendo mayor con respecto al porcentaje detectado por citogenética convencional, por lo que los aCGH o los microarreglos no comparativos se están convirtiendo en el estudio de primera elección para pacientes con

Cuadro 2. Reportes de estudios realizados en pacientes con RM por aCGH

Referencia Casos estudiados
Frecuencia de alterados

Porcentaje de detección

\begin{tabular}{lccc} 
Rosenberg et al. $^{29}$ & 81 & 13 de 81 & 16.00 \\
\hline Lugtenberg et al. $^{10}$ & 40 & 3 de 40 & 7.50 \\
\hline Friedman et al. $^{30}$ & 100 & 11 de 100 & 11.00 \\
\hline Krepischi-Santos et al. $^{31}$ & 95 & 16 de 95 & 17.00 \\
\hline Uwineza et al. $^{32}$ & 50 & 13 de 50 & 26.00 \\
\hline Choucair et al. $^{33}$ & 149 & 18 de 149 & 12.10 \\
\hline Yokoyama et al. $^{34}$ & 152 & 32 de 152 & 21.05
\end{tabular}


DI idiopática, alteraciones del espectro autista y múltiples malformaciones congénitas.

El establecer un diagnóstico de certeza, permitirá ofrecer algún tipo de manejo y sobre todo un asesoramiento genético adecuado para toda la familia, ya que posibilita al genetista estimar un riesgo de recurrencia más certero para futuros embarazos. Para los padres, esto generalmente disminuye su ansiedad, ya que en la mayoría de los casos el evento es de novo y la pareja tendrá un riesgo de recurrencia muy bajo.

\section{REFERENCIAS}

1. Rodríguez-Revenga Bodi L, Madrigal-Bajo I, Milà-Racasens M. Genetic mental retardation. Rev Neurol. 2006;43 (S1):S181-6.

2. American Psychiatric Association. American Psychiatric Association: Diagnostic and Statistical Manual of Mental Disorders. 5th ed. Arlington: 2013.

3. American Association on Mental Retardation. Mental retardation: definition, classification, and systems of supports, 10th ed. Washington DC: American Association on Mental Retardation, 2002.

4. WHO. The ICD-10 classification of mental and behavioural disorders. Geneva: WHO, 1992.

5. Rudolph A, Kamei R, Overby Kim. Rudoph's Fundamentals of Pediatrics. 3a Ed., Estados Unidos. Mc-Graw Hill 2002.

6. McLaren J, Bryson SE. Review of recent epidemiological studies of mental retardation: prevalence, associated disorders, and etiology. Am J Ment Retard 1987; 92: 243-54.

7. Stevenson RE. Human malformations and related anomalies. En Stevenson RE, Hall JG. Human malformations and related anomalies. New York, Oxford University Press. 2006;1:3-13.

8. Firth HV, Hurst JA. Approach to the consultation with a child with dysmorphism, congenital malformation, or developmental delay. Oxford Desk Reference - Clinical Genetics. Oxford University Press. 2005:4-5.

9. Lugtenberg D, de Brouwer APM, Kleefstra T, Oudakker AR, Frints SGM, Schrander-Stumpel CTRM, et al. Chromosomal copy number changes in patients with non-syndromic $X$ linked mental retardation detected by array CGH. J Med Genet 2006;43:362-370.

10. Castellví-Bel S, Milà M. Genes responsible for nonspecific mental retardation. Mol Genet Metab. 2001;72(2):104-8.

11. Stankiewicz P, Beaudet AL. Use of array CGH in the evaluation of dysmorphology, malformations, developmental delay, and idiopathic mental retardation. Curr Opin Genet Dev. Jun 2007;17(3):182-92.
12. Eirís-Puñal J. Aportación de la genética y de los estudios neurometabólicos al diagnóstico del retraso mental. Rev Neurol 2006;43(Supl. 1):S177-S180.

13. Tjio JH, Levan A. The chromosome number of man. Hereditas 1956;42:1-6.

14. Lejeune J, Gautier M, Turpin R. Études des chromosomes somatiques de neuf enfants mongoliens. C R Acad Sci.1959;248(11):1721-1722.

15. de Ravel TJ, Devriendt K, Fryns JP, Vermeesch JR. What's new in karyotyping? The move towards array comparative genomic hybridisation (CGH). Eur J Pediatr. 2007;166(7):637-43.

16. $\mathrm{Xu} \mathrm{J}, \mathrm{Chen} Z$. Advances in molecular cytogenetics for the evaluation of mental retardation. Am J Med Genet C Semin Med Genet 2003; 117:15-24.

17. Shevell M, Ashwal S, Donley D, Flint J, Gingold M, Hirtz D, et al. Quality Standards Subcommittee of the American Academy of Neurology; Practice Committee of the Child Neurology Society. Practice parameter: evaluation of the child with global developmental delay: report of the Quality Standards Subcommittee of the American Academy of Neurology and The Practice Committee of the Child Neurology Society. Neurology. 2003;60:367-80.

18. Van Karnebeek CD, Jansweijer MC, Leenders AG, Offringa $M$, Hennekam RC. Diagnostic investigations in individuals with mental retardation: a systematic literature review of their usefulness. Eur J Hum Genet. 2005;13:6-25.

19. Guitart-Feliubadaló M, Brunet-Vega A, Villatoro-Gómez S, Baena-Díez N, Gabau-Vila E. Causas cromosómicas que originan el retraso mental: alteraciones cromosómicas diagnosticables en el paciente. Rev Neurol. 2006;42(Supl 1):S21-S26

20. Salinas-Toldo S, Lampel S, Stilgenbauer S, Nickolenko J, Benner A, Döhner H, Cremer T, Lichter P. Matrix-based comparative genomic hybridization: biochips to screen for genomic imbalances. Genes Chromosomes Cancer 1997;20(4):399-407.

21. Pinkel D, Segraves R, Sudar D, Clark S, Poole I, Kowbel D, et al. High resolution analysis of DNA copy number variation using comparative genomic hybridization to microarrays. Nature Genet .1998;20(2):207-211.

22. Bejjani BA, Theisen AP, Ballif BC, Shaffer LG. Array-based comparative genomic hybridization in clinical diagnosis. Expert Rev Mol Diag. 2005; 5(3):421-429.

23. Feero WG, Guttmacher AE, Collins FS. Genomic Medicine - An Update Primer. N Engl J Med. 2010;362:2001-11.

24. Van Karnebeek CD, Jansweijer MC, Leenders AG, Offringa $M$, Hennekam RC. Diagnostic investigations in individuals with mental retardation: a systematic literature review of their usefulness. Eur J Hum Genet. 2005;13:6-25.

25. Shaffer LG, Bejjani BA, Torchia B, Kirkpatrick S, Coppinger $\mathrm{J}$, Blallif $\mathrm{BC}$. The identification of microdeletion syndromes and other chromosome abnormalities: cytogenetic methods of the past, new technologies for the future. Am J Med Genet Part C Semin Med Genet. 2007;145c:335-345. 
Yokoyama-Rebollar E et al. Abordaje de pacientes con DI y malformaciones

26. Ballif BC, Sulpizio SG, Lloyd RM, Minier SL, Theisena A, Bejjani BA, Shaffer LG. The clinical utility of enhaced subtelomeric coverage in array CGH. Am J Med Genet Part A. 2007;1143a:1850-1857.

27. Shaffer LG, Ballif BC, Theisen A, Rorem E, Bejjani BA, Torchia BA. In the middle of it all: a centered approach to chromosome analysis. Expert Opin Med Diagn. 2008;2(2):1-8.

28. Qiao Y, Badduke C, Mercier E, Lewis SM, Pavlidis P, RaicanSeparovic E. miRNA and miRNA target genes in copy number variations occurring in individuals with intellectual disability. BMC Genomics. 2013;14:544.

29. Rosenberg C, Knijnenburg J, Bakker E, Vianna-Morgante AM, Sloos W, Otto PA, et al. Array-CGH detection of micro rearrangements in mentally retarded individuals: clinical significance of imbalances present both in affected children and normal parents. J Med Genet. 2006;43(2):180-6.

30. Friedman JM, Baross A, Delaney AD, Ally A, Arbour L, Armstrong $L$, et al. Oligonucleotide microarray analysis of genomic imbalance in children with mental retardation. Am J Hum Genet. 2006;79(3):500-13.
31. Krepischi-Santos AC, Vianna-Morgante AM, Jehee FS, Passos-Bueno MR, Knijnenburg J, Szuhai K, et al. Wholegenome array-CGH screening in undiagnosed syndromic patients: old syndromes revisited and new alterations. Cytogenet Genome Res. 2006;115(3-4):254-61.

32. Uwineza A, Caberg JH, Hitayezu J, Hellin AC, Jamar M, Dideberg V, Rusingiza EK, Bours V, Mutesa L. Array-CGH analysis in Rwandan patients presenting development delay/inteIlectual disability with multiple congenital anomalies. BMC Med Genet. 2014;15:79. doi: 10.1186/1471-2350-15-79.

33. Choucair N, Ghoch JA, Corbani S, Cacciagli P, Mignon-Ravix C, Salem N, et al. Contribution of copy number variants (CNVs) to congenital, unexplained intellectual and developmental disabilities in Lebanese patients. Mol Cytogenet. 2015;8:26. doi: 10.1186/s13039-015-0130-y.

34. Yokoyama-Rebollar E. Detección de alteraciones cromosómicas por microarreglos-cgh y su relación en pacientes que cursan con retraso mental o retraso psicomotor idiopático. 2016. http://132.248.9.195/ptd2016/febrero/503041247/ Index.html 